\title{
Imprecise input data and Value at Risk estimation
}

\author{
Michal Holčapek, Tomáš Tichý
}

\begin{abstract}
During last decades the stochastic simulation approach, both via MC and QMC has been vastly applied and subsequently analyzed in almost all branches of science. Very nice applications can be found in areas that rely on modeling via stochastic processes, such as finance. However, since financial quantities -- opposed to natural processes -- depend on human activity, their modeling is often very challenging. Many scholars therefor suggest to specify some parts of financial models by means of fuzzy set theory. In this paper we formulate a fuzzystochastic model to be solved by Monte Carlo simulation. The application possibility is shown on the case of Value at Risk estimation of a single position, though it can be easily generalized to deal with more complex problem.
\end{abstract}

Keywords - fuzzy random variable, fuzzy quantile, fuzzy stochastic process, Value at Risk estimation

\section{Introduction}

During last decades the stochastic simulation approach, both via MC and QMC has been vastly applied and subsequently analyzed in almost all branches of science. Very nice applications can be found in areas that rely on modeling via stochastic processes. For example, in finance it is usually assumed that returns of almost all assets - riskless bond is an exception - can be described by stochastic differential equation based on random term with a suitable probability distribution. However, opposed to processes in nature financial processes crucially depend on human behavior and are rather imprecise (vague) than random. Modeling is therefore more challenging. More importantly, it is often difficult to provide reliable parameter estimates for particular models.

As an alternative, many researchers suggest to specify a financial model or at least its parameters as a fuzzy number, ie. instead of real variables with a suitable distribution function we assume potentially infinite number of intervals, each with its membership function as given by particular $\alpha$ (see Section 2 for more detailed description).

The application of fuzzy set theory in finance usually restricts to basic "analytic" operations with fuzzy and crisp inputs and includes Black and Scholes or binomial model type option pricing problem (see eg. Simonelli (2001) or Zmeškal (2001)), Value at Risk estimation (Zmeškal, 2005), portfolio

Michal Holčapek, Tomáš Tichý

Department of Finance

Technical University Ostrava

Czech Republic selection process (Tanaka et al., 2000), time series analysis ( $\mathrm{Li}$ and Cheng, 2006) or insurance problems (Huang et al, 2008). However, it is well known that many problems in finance cannot be solved analytically even if simple (geometric) Brownian motion is assumed (eg. exotic option valuation). It is therefore surprising that there are just a few papers on MC simulation of fuzzy random models and usually with quite inadequate treatment of the simulation approach (see eg. Nowak and Romaniuk, 2010).

In our previous research, we have focused on option pricing (Holčapek and Tichý, 2011, 2013). By contrast, in this paper we analyze the impact of various input data on Value at Risk estimation. In the following sections we utilize recent knowledge of fuzzy numbers and their approximation and suggest simulation approach to their modeling. We also provide distinct types of the results after fuzzyfication - due to the $\alpha$-cut and defuzzified quantile.

\section{LU-fuzzy numbers}

Let $\mathrm{R}$ denotes the set of real numbers. A fuzzy number is usually called a mapping $A: \mathrm{R} \rightarrow[0,1]$ which is normal (i.e. there exits an element $x_{0}$ such that $\left.A\left(x_{0}\right)=1\right)$, convex (i.e. $A(\lambda$ $x+(1-\lambda) y) \geq \min (A(x), A(y)$ for any $x, y$ in $\mathrm{R}$ and $\lambda$ in $[0,1])$, upper semicontinuous and $\operatorname{supp} A$ is bounded, where sup $A=$ $\operatorname{cl}\{x$ in $\mathrm{R} \mid A(x)>0\}$ and $c l$ is the closure operator (see Klir and Yuan, 1995 and Dubois and Prade, 1978). The most popular models of fuzzy numbers are the triangular and trapezoidal models investigated by Dubois and Prade (1980). Their popularity follows from the simple (fuzzy) calculus as addition or multiplication of fuzzy numbers which can be established for them. This is also a reason why we can find many recent papers on the approximation of fuzzy numbers by the mentioned models (see e.g. Ban (2009a,b) and the references therein).

In order to model fuzzy numbers we will use a more advanced model of fuzzy numbers based on the interpolation of given knots using rational splines that was proposed by Guerra and Stefanini (2005) and developed in Stefanini et al. (2006). This model generalizes the triangular fuzzy numbers and gives a broad variety of shapes enabling more precise representation of fuzzy real data, nevertheless, the calculus is still very simple.

It is well known that each fuzzy number has a representation using $\alpha$-cuts. Recall that $\alpha$-cut of a fuzzy number $A$ is the common set $A_{\alpha}=\{x$ in $\mathrm{R} \mid A(x) \geq \alpha\}$ and

$$
A(x)=\bigvee_{\substack{\alpha \in 0,1 \mid \\ x \in A,}} a .
$$


Since the fuzzy numbers are upper semicontinuous real functions, then each $\alpha$-cut may be replaced by its endpoints, say $u^{-}(\alpha)$ for the left endpoint and $u^{+}(\alpha)$ for the right endpoint. Hence, each fuzzy number can be completely represented by two functions $u, v:[0,1] \rightarrow \mathrm{R}$ such that

(i) $u^{-}$is a bounded monotonic non-decreasing function which is left-continuous on $] 0,1]$ and the right-continuous for $\alpha=0$,

(ii) $\mathrm{u}^{+}$is a bounded monotonic non-increasing function which is left-continuous on ]0,1] and the right-continuous for $\alpha=0$,

(iii) $u^{-}(\alpha) \leq u^{+}(\alpha)$ for any $\alpha$ in $[0,1]$.

The arithmetic operations between two fuzzy numbers $A$ and $B$ represented by pairs of functions $\left(u_{A}^{-}, u_{A}^{+}\right)$and $\left(u_{B}^{-}\right.$, $u_{B}^{+}$), respectively, can be introduced using a suitable manipulation of the functions $u$ and $v$. For example, $A+B$ can be simply obtained by $\left(u_{A}^{-}+u_{B}^{-}, u_{A}^{+}+u_{B}^{+}\right)$. For further definitions of arithmetic operations, we refer to Stefanini et al. (2006). Since the modeling of fuzzy numbers and the manipulation with them is not so simple in general, we use the parametric representation of fuzzy numbers proposed by Stefanini et al. (2006).

A piecewise rational cubic Hermite parametric function $P$ in $\mathrm{C}^{1}\left[\alpha_{0}, \alpha_{n}\right]$, with parameters $v_{i}, w_{i}, i=0, \ldots, n-1$, is defined for $\alpha$ in $\left[\alpha_{i}, \alpha_{i+1}\right], i=0, \ldots, n-1$ by

$$
\begin{aligned}
P(\alpha)= & P_{i}\left(\alpha, v_{i}, w_{i}\right)= \\
& \left((1-\theta)^{3} f_{i}+\theta(1-\theta)^{2}\left(v_{i} f_{i}+h_{i} d_{i}\right)+\right. \\
& \left.\theta^{2}(1-\theta)\left(w_{i} f_{i+1}-h_{i} d_{i+1}\right)+\theta^{3} f_{i+1}\right) / \\
& \left((1-\theta)^{3}+v_{i} \theta(1-\theta)^{2}+\right. \\
& w_{i} \theta^{2}(1-\theta)+\theta^{3},
\end{aligned}
$$

where the notations $f_{i}$ and $d_{i}$ are, respectively, the real data values and the first derivative values (slopes) at the knots $\alpha_{0}<$ $\alpha_{1}<\ldots<\alpha_{n}, h_{i}=\alpha_{i+1}-\alpha_{i}, \theta=\left(\alpha-\alpha_{\mathrm{i}}\right) / h_{i}$ and $v_{i}, w_{i} \geq 0$. The parameters $v_{i}$ and $w_{i}$ are called the tension parameters. In the case $v_{i}=w_{i}=3$, we obtain the ordinary cubic spline. For details, we refer to Gregory (1986), Sarfraz (2000). One can see that each parametric function $P$ in $C^{1}\left[\alpha_{0}, \alpha_{n}\right]$ may be expressed in the matrix form consisting of parameters

$$
\mathbf{P}=\left(\begin{array}{c}
\mathbf{f} \\
\mathbf{d} \\
\mathbf{v} \\
\mathbf{w}
\end{array}\right)=\left(\begin{array}{cccc}
f_{\alpha_{0}} & \ldots & f_{\alpha_{n-1}} & f_{\alpha_{n}} \\
d_{\alpha_{0}} & \ldots & d_{\alpha_{n-1}} & d_{\alpha_{n}} \\
v_{\alpha_{0}} & \cdots & v_{\alpha_{n-1}} & 0 \\
w_{\alpha_{0}} & \cdots & w_{\alpha_{n-1}} & 0
\end{array}\right)
$$

for a partition $\alpha_{0}<\ldots<\alpha_{n}$ of the interval $\left[\alpha_{0}, \alpha_{n}\right]$. We use 0 for $v_{n}$ and $w_{n}$, since these parameters do not play any role in the derivation of the function $P$. We will write $P=\mathbf{P}$, if $P$ is determined by the matrix $\mathbf{P}$.

Now we may define so-called LU-fuzzy number introduced in Guerra and Stefanini (2005). Note that our definition is slightly different than the original one, but the idea remains the same.

Definition 1. A fuzzy number $A$ determined by $\left(u^{-}, u^{+}\right)$is an $L U$-fuzzy number, if there exist a partition $0=\alpha_{0}<\ldots<\alpha_{n}=1$ and $4 n+1$ matrices $\mathbf{U}^{-}$and $\mathbf{U}^{+}$such that

(i) $u^{-}=\mathbf{U}^{-}$and $u^{+}=\mathbf{U}^{+}$,

(ii) $f\left(\alpha_{i+1}\right) \geq f^{-}\left(\alpha_{i}\right)$ and $f^{+}\left(\alpha_{i+1}\right) \leq f^{+}\left(\alpha_{i}\right)$ for any $i=0, \ldots$, $n$,

(iii) $d\left(\alpha_{i}\right) \geq 0$ and $d^{+}\left(\alpha_{i}\right) \leq 0$ for any $i=0, \ldots, n$,

(iv) $f^{-}\left(\alpha_{n}\right)=f^{+}\left(\alpha_{n}\right)$,

(v) $v^{-}\left(\alpha_{i}\right), v^{+}\left(\alpha_{i}\right), w^{-}\left(\alpha_{i}\right), w^{+}\left(\alpha_{i}\right) \geq 0$ for any $i=0, \ldots, n-$ 1 and $v^{-}\left(\alpha_{n}\right), v^{+}\left(\alpha_{n}\right), w^{-}\left(\alpha_{n}\right), w^{+}\left(\alpha_{n}\right)=0 \$$,

(vi) if $f\left(\alpha_{i}\right)=f\left(\alpha_{i+1}\right)$ (or $\left.f^{+}\left(\alpha_{i}\right)=f^{+}\left(\alpha_{i+1}\right)\right)$, then $d\left(\alpha_{i}\right)=$ $\bar{d}\left(\alpha_{i+1}\right)=v^{-}\left(\alpha_{i}\right)=w^{-}\left(\alpha_{i}\right)=0\left(\right.$ or $d^{+}\left(\alpha_{i}\right)=d^{+}\left(\alpha_{i+1}\right)=$ $\left.v^{+}\left(\alpha_{i}\right)=w^{+}\left(\alpha_{i}\right)=0\right)$.

The set of all LU-fuzzy numbers will be denoted by $F_{L U}$.

Note that one can define a fuzzy interval, if the equality in (iv) is replaced by $f^{-}\left(\alpha_{n}\right)<f^{+}\left(\alpha_{n}\right)$. Further, the last condition is more or less technical and the simple consequence of this is $u^{-}(\alpha)=f^{-}\left(\alpha_{i}\right)=f\left(\alpha_{i+1}\right)$ for any $\alpha$ in $\left[\alpha_{i}, \alpha_{i+1}\right]$. For example, a real number $\$ r \$$ can be naturally expressed as the LU-fuzzy number with the matrices

$$
\mathbf{U}^{-}=\mathbf{U}^{+}=\left(\begin{array}{ccc}
r & \ldots & r \\
0 & \ldots & 0 \\
0 & \ldots & 0 \\
0 & \ldots & 0
\end{array}\right) .
$$

Obviously, higher number $n$ may be used for a better approximation of a complex fuzzy number obtained, for example, from the data observation. A simplification of LUfuzzy numbers can be done by the determination of the vectors $\mathbf{v}^{ \pm}$and $\mathbf{w}^{ \pm}$from the $\mathbf{f}^{ \pm}$and $\mathbf{d}^{ \pm}$. Here, we use notation \pm to express an assertion for - and + together. This is mainly profitable when the arithmetic operations are defined. In this work, we will also use a global monotonicity setting (see Stefanini et al., 2006):

$$
v_{i}^{\mp}=w_{i}^{\mp}= \begin{cases}\frac{d_{i+1}^{\mp}+d^{\mp}}{f_{i+1}^{\mp}-f_{i}^{\mp}}, & f_{i+1}^{\mp} \neq f_{i}^{\mp} ; \\ 0, & \text { otherwise. }\end{cases}
$$

One can simply check that formulation above is correct and we obtain $v_{i}^{ \pm}, \mathrm{w}_{i}^{ \pm} \geq 0$.

Example 1. Let us consider

$$
\mathbf{U}^{-}=\left(\begin{array}{cccc}
4 & 4.5 & 5 & 6.5 \\
2 & .1 & .1 & 25 \\
2 & 2 & 3 & 0 \\
3 & 3 & 3 & 0
\end{array}\right)
$$

and 
Proc. of the Third Intl. Conf. on Advances in Economics, Management and Social Study - EMS 2015

Copyright (C) Institute of Research Engineers and Doctors, USA .All rights reserved.

ISBN: 978-1-63248-058-3 doi: 10.15224/ 978-1-63248-058-3-56

$$
\mathbf{U}^{+}=\left(\begin{array}{cccc}
9 & 7.5 & 7 & 6.5 \\
-.1 & -.1 & -.1 & -25 \\
2 & 2 & 3 & 0 \\
3 & 3 & 3 & 0
\end{array}\right)
$$

The LU-fuzzy number about 6.5 determined by $\mathbf{U}^{+}$and $\mathbf{U}^{-}$ is illustrated in Fig. 1.

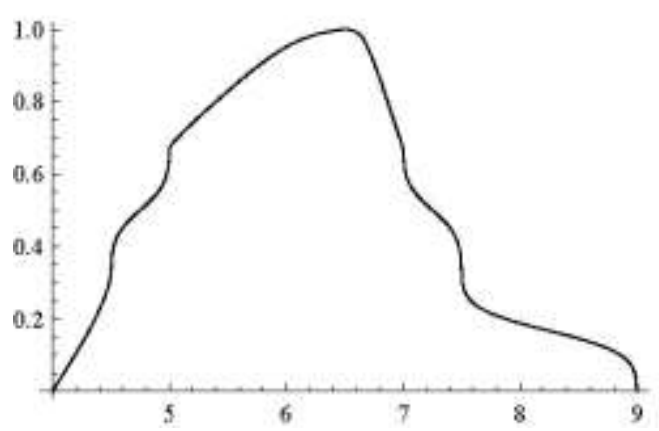

Figure 1: Fuzzy number determined by the matrices $\mathbf{U}^{+}$and $\mathbf{U}^{-}$from Ex. 1.

\section{Fuzzy random variable}

We follow the approach to fuzzy random variable proposed by Kwakernaak $(1978,1979)$ and later formalized in a clear way by Kruse and Meyer (1987). In this approach, a fuzzy random variable is viewed as a fuzzy perception/observation/report of a classical real-valued random variable. The model is stated as follows (we use the LU-fuzzy numbers to represent the values of fuzzy random variables).

Definition 2. Given a probability space $(\Omega, A, \mathrm{P})$, a mapping $\mathrm{X}: \Omega \rightarrow F_{L U}(v, w)$ is said to be a fuzzy random variable if for all $\alpha$ in $[0,1]$ the two real-valued mappings inf $\mathrm{X}_{\alpha}: \Omega \rightarrow \mathrm{R}$, sup $\mathrm{X}_{\alpha}: \Omega \rightarrow \mathrm{R}$ (defined so that for all $\omega$ in $\Omega$ we have that $\mathrm{X}_{\alpha}(\omega)=\left[\inf \mathrm{X}(\omega)_{\alpha}, \sup \mathrm{X}(\omega)_{\alpha}\right]$ ) are real-valued random variables.

Since each LU-fuzzy number is representable by matrices $\mathrm{U}^{-}$and $\mathrm{U}^{+}$, we can define fuzzy random variable using random matrices as follows.

Definition 3. Given a probability space $(\Omega, A, \mathrm{P})$, a mapping $\mathrm{X}: \Omega \rightarrow F_{L U}(v, w)$ is said to be a fuzzy random variable (FRV) if there exist a partition $0=\alpha_{0}<\ldots<\alpha_{n}=1$ of the interval $[0,1]$ and mappings

$$
\mathrm{F}^{-}, \mathrm{F}^{+}, \mathrm{D}^{-}, \mathrm{D}^{+}: \Omega \rightarrow \mathrm{R}^{n+1}
$$

such that $p_{i} \circ \mathrm{F}^{-}, p_{i} \circ \mathrm{F}^{+}, p_{i} \circ \mathrm{D}^{-}, p_{i} \circ \mathrm{D}^{+}$, where $p_{i}$ denotes $i$-th projection, are real-valued random variables for any $i=1, \ldots, n$ +1 and $X(\omega)$ is determined by

$$
\begin{aligned}
& \mathbf{U}^{-}(\omega)=\left(\begin{array}{l}
\mathbf{F}^{-}(\omega) \\
\mathbf{D}^{-}(\omega)
\end{array}\right)= \\
& =\left(\begin{array}{lll}
p_{1} \circ \mathbf{F}^{-}(\omega) & \ldots & p_{n+1} \circ \mathbf{F}^{-}(\omega) \\
p_{1} \circ \mathbf{D}^{-}(\omega) & \ldots & p_{n+1} \circ \mathbf{D}^{-}(\omega)
\end{array}\right) \\
& \mathbf{U}^{+}(\omega)=\left(\begin{array}{lll}
\mathbf{F}^{+}(\omega) \\
\mathbf{D}^{+}(\omega)
\end{array}\right)= \\
& =\left(\begin{array}{lll}
p_{1} \circ \mathbf{F}^{+}(\omega) & \ldots & p_{n+1} \circ \mathbf{F}^{+}(\omega) \\
p_{1} \circ \mathbf{D}^{+}(\omega) & \ldots & p_{n+1} \circ \mathbf{D}^{+}(\omega)
\end{array}\right) .
\end{aligned}
$$

Definition 3. We say that two FRVs $X$ and $Y$ are independent (identically distributed), if $p_{i} \circ \mathrm{F}_{X}^{-}, p_{i} \circ \mathrm{F}_{X}{ }^{+}, p_{i} \circ \mathrm{D}_{X}^{-}, p_{i} \circ \mathrm{D}_{X}{ }^{+}$and $p_{i} \circ \mathrm{F}_{Y}^{-}, p_{i} \circ \mathrm{F}_{Y}^{+}, p_{i} \circ \mathrm{D}_{Y}^{-}, p_{i} \circ \mathrm{D}_{Y}^{+}$are independent (identically distributed), respectively, for any $i=1, \ldots, n+1$.

Note that using the interpolation we obtain random variables $u^{-}(\alpha)$ and $\mathrm{u}^{+}(\alpha)$ under the probability space $(\Omega, A, \mathrm{P})$. Hence, the FRVs introduced above are FRVs in the KruseMeyer sense.

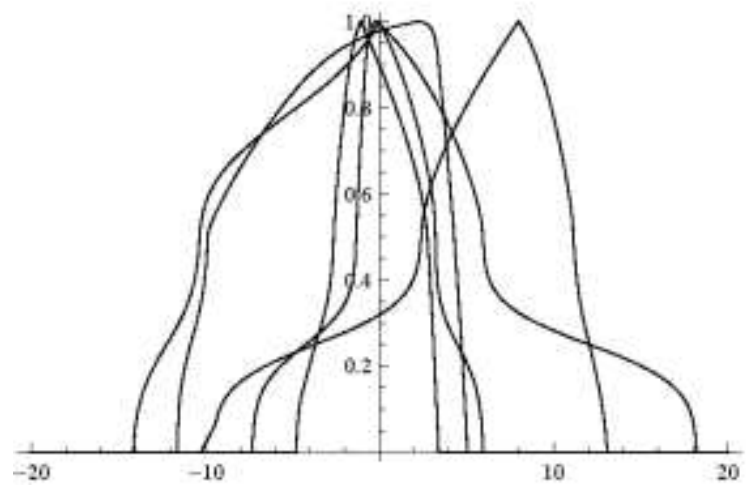

Figure 2: Pseudorandom LU-fuzzy numbers

On Fig. 2 we can see five pseudo-randomly generated LUfuzzy numbers defined under the normal distribution (the kernels, i.e., the points with the membership degree equal to 1 , are determined from $N(0,4)$, further values are determined in such way that the difference between $f\left(\alpha_{i}\right)$ and $f\left(\alpha_{i+1}\right)$ is a random value from $N(0,2)$ ).

\section{Iv. Financial models with imprecise data}

Finally, in this section we apply the fuzzy theory that give us the possibility to deal with imprecisely specified input data (fuzzy data). For this purpose we assume modified standard market model, ie. Brownian motion, with crisp volatility parameter replaced by fuzzy parameter.

\section{A. Standard market model}

Assume a standard market model, ie. Brownian motion with drift $\mu$ and standard deviation $\sigma$.

$$
X(t)=\mu t+\sigma \sqrt{ } t \varepsilon,
$$


where $e$ is from $N[0,1]$ (ie. it is of standard normal distribution). This is a standard market model for (log-returns) returns of financial asset prices, such as stocks, commodities or exchange rates. Since it can be difficult to obtain a reliable estimate of standard deviation $\sigma$, we can define it as an LUfuzzy number:

$$
X(t)=\mu t+\sigma_{L U} \sqrt{ } t \varepsilon,
$$

There exist several ways how $\sigma_{L U}$ can be obtained. For example, we can split historical time series into several subperiods and obtain several distinct volatilities $\sigma$, which can be related to particular stage of the economy or risk attitude of investors. Alternatively, for asset prices without long history, we can take volatility estimates of similar securities.

\section{B. VaR estimation}

In finance, it is often important to know the quantiles, ie. what is the limit result (return, loss, price, etc.) for a given probability, or alternatively, what is the probability of a given limited value of return or price. The estimated quantiles are commonly used within riskmangement (internal, capital regulation, etc.) and are referred to as Value at Risk (VaR). Next, the quantiles can be used to calculation of expected shortfalls or even option price.

As an illustration let us suppose time horizon of one year (which might be related to capital requirements in insurance sector), drift term of $10 \%$ and LU-stochastic volatility built up over $30 \%$ (ie. the most common value).

First, on Fig. 3 we present estimation of various quantiles for all $\alpha$-cuts, i.e. we can see how is the quantile spread for particular membership levels. In particular, we assume quantiles for the following probabilities: 0.001, 0.01, 0.05, 0.1. These quantiles can be easily transferred into Value at Risk by inverting the sign. Thus, assuming $\alpha$-cut of 0.95 , that is, very good knowledge about the input volatility, the estimation of VaR would be:

$$
\begin{gathered}
\operatorname{VaR}(0.001)=(-12.3333,-7.3838), \\
\operatorname{VaR}(0.01)=(-9.643,-5.96852), \\
\operatorname{VaR}(0.05)=(-7.36809,-4.77178), \\
\operatorname{VaR}(0.1)=(-6.18046,-4.14701) .
\end{gathered}
$$

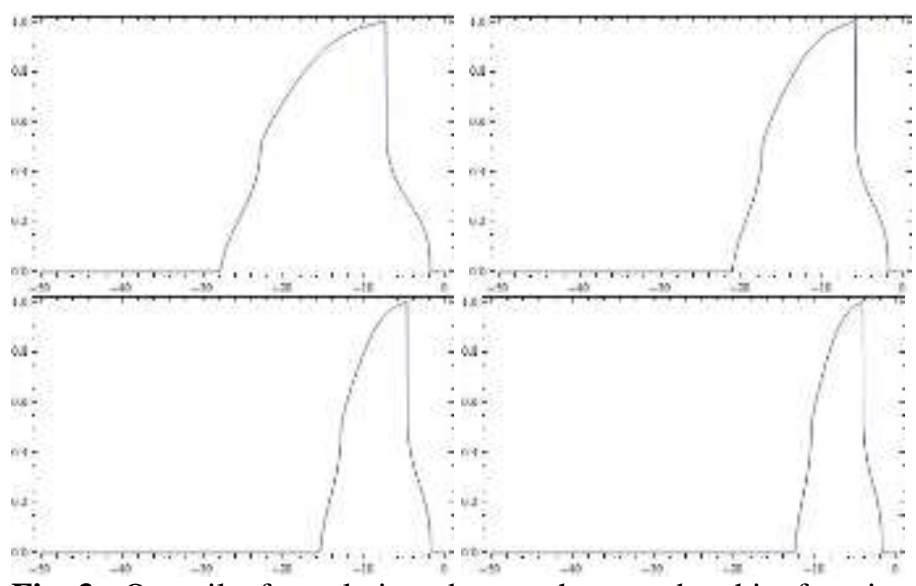

Fig 3: Quantile formulation due to the membership function assuming four distinct probability levels $(q=0.001, q=0.01, q$ $=0.05$, and $q=0.1$ )

As an alternative form of presentation we can consider the projection into $2 \mathrm{D}$ spectrum. Such projection can give us simple picture about both, the quantile estimation and membership function. In Fig. 4 we provide the results assuming the same input as above (left), as well as the fuzzydistribution for volatility cut by $50 \%$ (right).
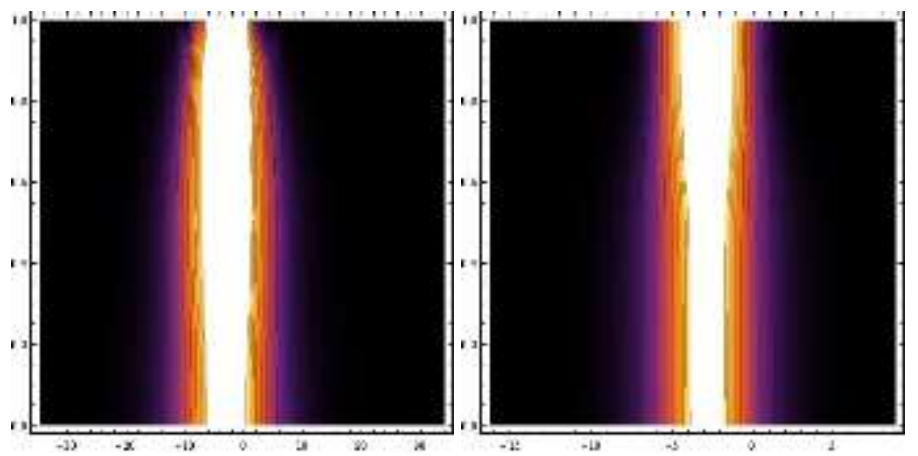

Fig. 4: $2 \mathrm{D}$ projection of original data and after rescaling of volatility by $50 \%$

\section{v. Conclusion}

Many issues of financial modeling and decision making require some knowledge about the future states. However, sometimes it is very difficult to get reliable parametrization of stochastic models. In this contribution we have formulated alternative approach to modeling of financial quantities based on fuzzy random values. This approach includes interval and crisp approach as a special case.

Suggested models of financial returns can be incorporated into classic problems of financial modeling, such as portfolio VaR estimation and option valuation. However, depending on the type of the problem, additional operations with LU-fuzzy numbers might be useful.

\section{Acknowledgment}

The research was supported through the Czech Science Foundation (GACR) under project 13-13142S and through SP2015/15, an SGS research project of VSB-TU Ostrava, and 
furthermore by the European Social Fund in the framework of CZ.1.07/2.3.00/20.0296.

\section{References}

[1] A. I. Ban. On the nearest parametric approximation of a fuzzy number revisited. Fuzzy Sets Syst. 160(21):3027-3047, 2009.

[2] A. I. Ban. Triangular and parametric approximations of fuzzy numbers inadvertences and corrections. Fuzzy Sets Syst., 160(21):3048-3058, 2009.

[3] R. Cont, P. Tankov. Financial Modelling with Jump Processes Chapman \& Hall/CRC press, 2004.

[4] D. Dubois, H. Prade. Operations on fuzzy numbers. Int. J. Syst. Sci., 9:613-626, 1978.

[5] D. Dubois, H. Prade. Fuzzy sets and systems. Theory and applications. Mathematics in Science and Engineering, Vol. 144. New York etc.: Academic Press. XVII, 393 p. 1980.

[6] J. A. Gregory. Shape preserving spline interpolation. Comput. Aided Design, 18:5357, 1986.

[7] M. L. Guerra, L. Stefanini. Approximate fuzzy arithmetic operations using monotonic interpolations. Fuzzy Sets Syst. 150(1):5-33, 2005

[8] M. Holcapek, T. Tichy. Option Pricing with fuzzy parameters via Monte Carlo simulation. Communications in Computer and Information Science 211(1), 25-33, 2011.

[9] M. Holčapek, T. Tichý. Imprecise input data and option valuation problem. 15th WSEAS International Conference on Mathematical and Computational Methods in Science and Engineering, pp 204-209. 2013.

[10] C.-S. Huang, Y.-J. Lin, C.-C. Lin. Evaluation Models for Choosing Insurance Policy Using the AHP, Fuzzy Logic, and Delphi Technique. Applied computer and applied computational Science 696-703, 2008.

[11] G.J. Klir, Bo Yuan. Fuzzy Sets and Fuzzy Logic: Theory and Applications. Prentice Hall, New Jersey, 1995.

[12] R. Kruse, K. D. Meyer. Statistics with vague data. Theory and Decision Library. Series B: Mathematical and Statistical Methods, 6. Dordrecht etc.: D. Reidel Publishing Company, a member of the Kluwer Academic Publishers Group. VII, 279 p, 1987.

[13] H. Kwakernaak. Fuzzy random variables - I. Definitions and theorems. Inf. Sci. 15:1-29, 1978.

[14] H. Kwakernaak. Fuzzy random variables - II. Algorithms and examples for the discrete case. Inf. Sci. 17:253-278, 1979.

[15] S.T. Li, Y.-C. Cheng. A Hidden Markov Modelbased Forecasting Model for Fuzzy Time Series. Proceedings of the 10th WSEAS International Conference on SYSTEMS 436-441, 2006

[16] P. Nowak, M. Romaniuk. Computing option price for Levy process with fuzzy parameters. European Journal of Operational Research 201, 206210, 2010.

[17] M. Sarfraz. A rational cubic spline for the visualization of monotonic data. Comput. Graphics 24:509-516, 2000.

[18] M.R. Simonelli. Fuzziness in valuing financial instruments by certainty equivalents. European Journal of Operational Research 135/2, 296-302, 2001.

[19] L. Stefanini, L. Sorini, M. L. Guerra. Parametric representation of fuzzy numbers and application to fuzzy calculus. Fuzzy Sets Syst. 157(18):2423-2455, 2006.

[20] H. Tanaka, P. Guo, I.B. Turksen. Portfolio selection based on fuzzy probabilities and possibility distributions. Fuzzy Sets and Systems 111, 387-397, 2000.

[21] Z. Zmeskal. Application of the fuzzystochastic methodology to appraising the firm value as a European calls option. European Journal of Operational Research 135 (2), 303-310, 2001.

[22] Z. Zmeskal. Value at risk methodology under soft conditions approach (fuzzystochastic approach). European Journal of Operational Research 161 (2), 337-347, 2005. 